\title{
Qubit vs observable resource trade offs in measurement-based quantum computation
}

\author{
Simon Perdrix \\ Leibniz Laboratory, 46 avenue Félix Viallet 38000 Grenoble, France
}

\begin{abstract}
Since quantum measurement is universal for quantum computation (Nielsen [3]), the minimization of the resources required for measurement-based quantum computation is a crucial point $([3,1,4,5,6])$. We have shown in $[6]$ that a set of observables composed of one two-qubit measurement and three one-qubit measurements form a universal set for quantum computation if one ancillary qubit as additional resource is available. This set is minimal in terms of two-qubit measurements, because at least one multi-qubit operation is needed to create entanglement, and also minimal in terms of ancillary qubits, because at least one ancillary qubit is required to simulate reversible transformations with projective measurements only. We discuss in this paper the required number of one-qubit measurements for a set of observables to be universal. A set composed of one two-qubit measurement and only two one-qubit measurements is proved universal, at the cost of a second ancillary qubit. Thus an additional ancillary qubit seems to be required to obtain a smaller universal set of measurements. One may conclude to a trade off between ancillary qubits and observables in measurement-based quantum computation.
\end{abstract}

\section{MEASUREMENT-BASED QUANTUM COMPUTATION}

The set of observables $\mathscr{O}_{1}=\left\{Z, X, \frac{1}{\sqrt{2}}(X-Y), Z \otimes X\right\}$ (where $X, Y$ and $Z$ are Pauli matrices) is universal for quantum computation (see [7]). This universality comes from the ability to simulate any quantum circuit based on the universal set of unitary transformations $\{H, T, C N o t\}$ (see [2] for details on quantum circuits and [7] for details on the notions of universalities and simulations).

$$
H=\frac{1}{\sqrt{2}}\left(\begin{array}{cc}
1 & 1 \\
1 & -1
\end{array}\right), T=\left(\begin{array}{cc}
1 & 0 \\
0 & e^{\frac{i \pi}{4}}
\end{array}\right), C N o t=\left(\begin{array}{llll}
1 & 0 & 0 & 0 \\
0 & 1 & 0 & 0 \\
0 & 0 & 0 & 1 \\
0 & 0 & 1 & 0
\end{array}\right)
$$

This simulation is based on a 3-level decomposition: steps of simulation of $U$, where a unitary transformation $U$ is simulated up to a Pauli operator (i.e. a step of simulation of $U$ on $|\phi\rangle$ produces $\sigma U|\phi\rangle$, where $\sigma$ is a Pauli operator); full simulation of $U$, which combines a step of simulation of $U$ with steps of simulation of Pauli operators; and simulation of a quantum circuit, which combines full simulations of unitary transformations.

\section{Step of simulation of unitary transformations}

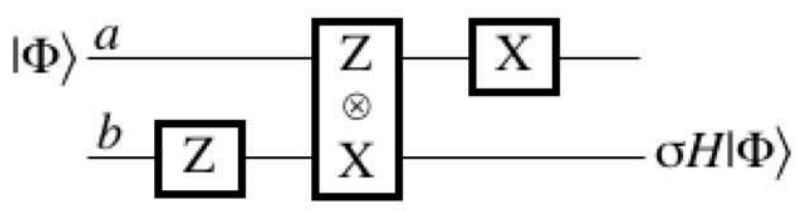

FIGURE 1. Step of simulation of $H$

For a given qubit $a$ and an ancillary qubit $b$, the sequence of measurements $\left\{Z^{(b)}, Z^{(a)} \otimes X^{(b)}, X^{(a)}\right\}$ (see fig. 1), simulates the application of the Hadamard transformation $H$ up to a Pauli operator which depends on the classical 
outcomes of the measurements.

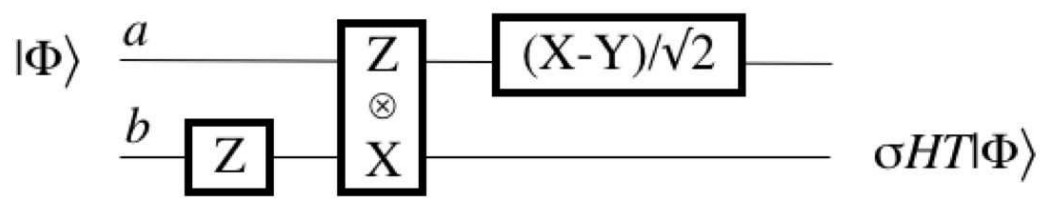

FIGURE 2. Step of simulation of $H T$

For a given qubit $a$ and an ancillary qubit $b$, the sequence of measurements $\left\{Z^{(b)}, Z^{(a)} \otimes X^{(b)},\left(\frac{X-Y}{\sqrt{2}}\right)^{(a)}\right\}$ (see fig. 2), simulates the application of the Hadamard transformation $H$ followed by the phase transformation $T$ up to a Pauli operator which depends on the classical outcomes of the measurements.

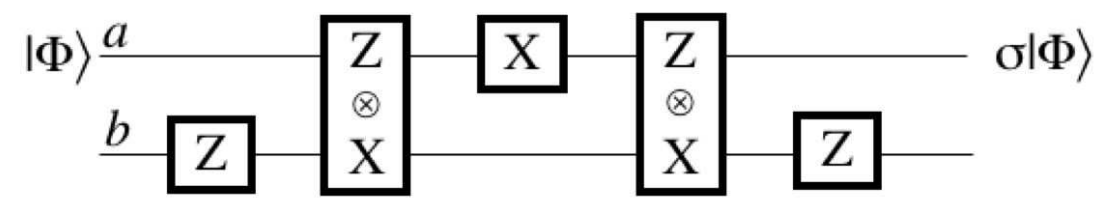

FIGURE 3. Step of simulation of Pauli operators

For a given qubit $a$ and an ancillary qubit $b$, the sequence of measurements $\left\{Z^{(b)}, Z^{(a)} \otimes X^{(b)}, X^{(a)}, Z^{(a)} \otimes X^{(b)}, Z^{(b)}\right\}$ (see fig. 3), introduces a Pauli operator which depends on the classical outcomes of the measurements. This sequence of measurement can be used to simulate any Pauli operator.

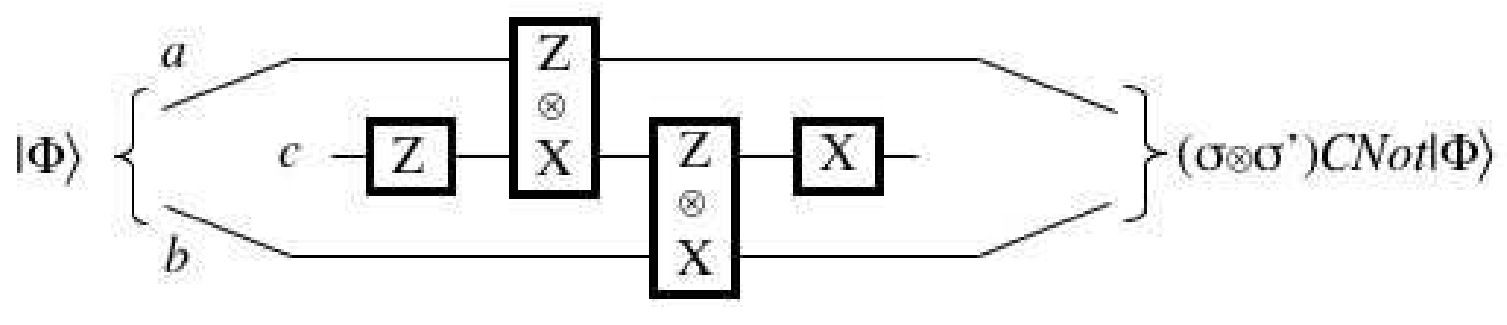

FIGURE 4. Step of simulation of $C N o t$

For a given 2-qubit register $a, b$ and one ancillary qubit $c$, the sequence of measurements $\left\{Z^{(c)}, Z^{(a)} \otimes X^{(c)}, Z^{(c)} \otimes\right.$ $\left.X^{(b)}, X^{(c)}\right\}$ (see fig. 4), simulates the 2-qubit unitary transformation CNot on the state $|\phi\rangle$ of $a, b$ up to a 2-qubit Pauli operator which depends on the classical outcomes of the measurements.

\section{Full simulation of unitary transformations}

For a given step of simulation of $U \in\{H, H T, C N o t\}$, the full simulation of $U$ is given by an automaton where each state encapsulates a step of simulation (fig. 5). This automaton is interpreted as follows: $U$ is simulated on a quantum state $|\phi\rangle$ so $\sigma U|\phi\rangle$ is obtained, where $\sigma$ is a Pauli operator. If $\sigma=I$ then the simulation is terminated, otherwise $\sigma$ is simulated. From this step of simulation, the state $\sigma^{\prime} \sigma \sigma U|\phi\rangle=\sigma^{\prime} U|\phi\rangle$ is obtained. If $\sigma^{\prime}=I$ the simulation is terminated, otherwise $\sigma^{\prime}$ is simulated, and so on. When the automaton halts, the state $U|\phi\rangle$ is obtained.

\section{Universality}

The model of quantum circuits based on the family of unitary transformations $\{H, H T, C N o t\}$ is quantum universal (see [2] for details on quantum circuits and [7] for details on the notions of universalities and simulations). Thus, since all unitary transformations can be fully simulated with measurements only, the model composed of automata where the states are steps of simulation is quantum universal. 

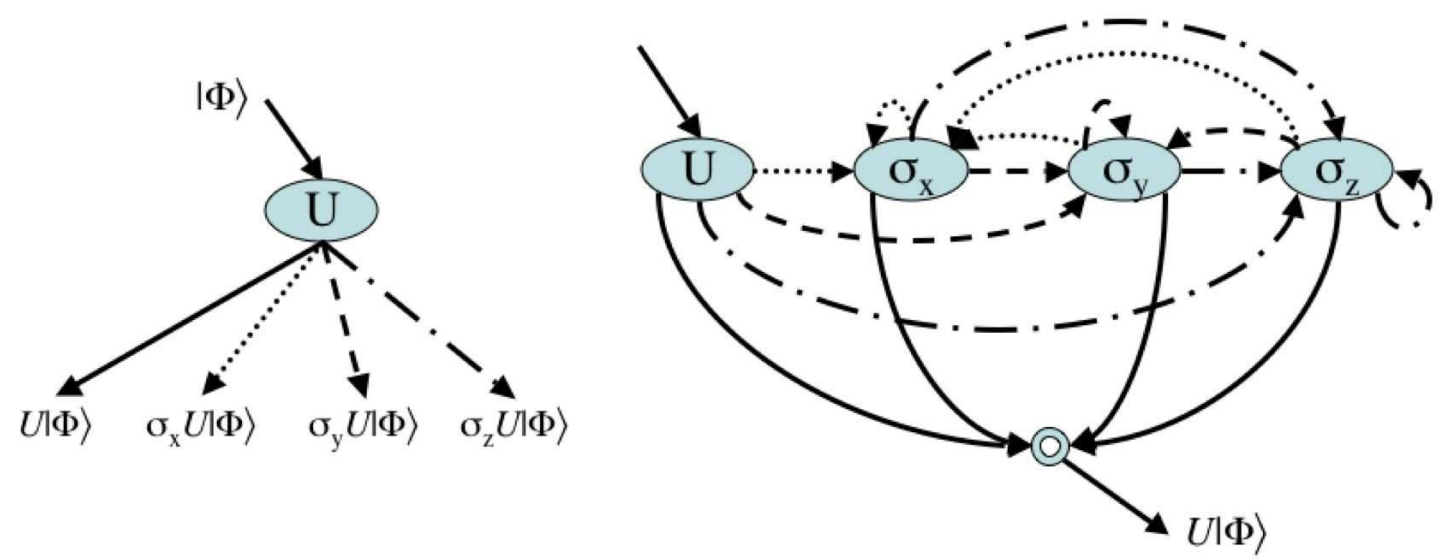

FIGURE 5. Left: a step of simulation of $U$ - Right: full simulation of $U$

\section{Resources}

For each step of simulation of $U \in\left\{H, H T, C N o t, \sigma_{x}, \sigma_{y}, \sigma_{z}\right\}$, only one ancillary qubit is needed. Since the simulation with measurements only of a given unitary transformation on an unknown state cannot be performed without ancillary qubit, the minimal resources necessary for quantum universality in terms of ancillary qubits are reached.

In terms of observables, since 1-qubit observables are not quantum universal, all universal families of observables contain at least one multi-qubit observable. Thus, since $\mathscr{O}_{1}=\left\{Z, X, \frac{1}{\sqrt{2}}(X-Y), Z \otimes X\right\}$ contains only one two-qubit observable, the minimal resources necessary for quantum universality in terms of the number of different multi-qubit observables, are reached.

However, the minimal number of 1-qubit observables is not reached.

\section{QUBIT VS OBSERVABLE RESOURCE TRADE OFFS}

In order to prove the universality of the set of observables $\mathscr{O}_{2}=\left\{Z, \frac{1}{\sqrt{2}}(X-Y), Z \otimes X\right\}=\mathscr{O}_{1}-\{X\}$, we introduce a sequence of measurements of $\mathscr{O}_{2}$ which simulates any measurement according to $X$.

For a given qubit $a$ and an ancillary qubit $b$, the sequence of measurements $\left\{Z^{(b)}, X^{(a)} \otimes Z^{(b)}\right\}$ (see fig. 6), simulates an $X$-measurement of the qubit $a$.
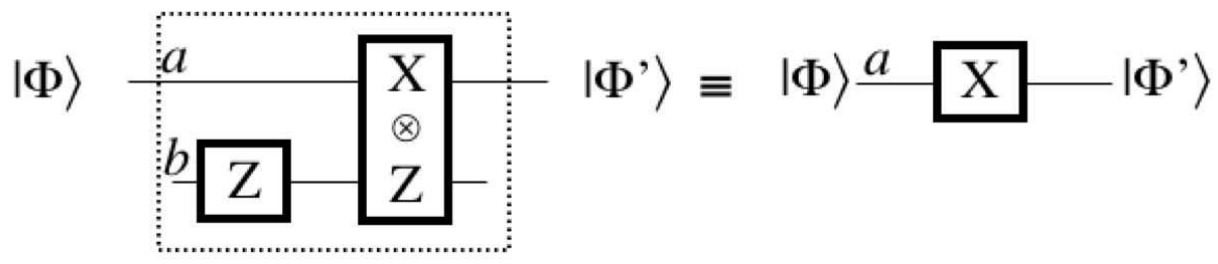

FIGURE 6. Simulation of an $X$-measurement

Each $X$-measurement of the steps of simulation presented in section 1 can be simulated using $Z$ - and $Z \otimes X$ measurements (see figure 6), thus the set of observables $\mathscr{O}_{2}=\left\{Z, \frac{1}{\sqrt{2}}(X-Y), Z \otimes X\right\}$ composed of one two-qubit measurement and only two one-qubit measurements is universal. However, this costs an additional ancillary qubit. As a consequence an additional ancillary qubit seems to be required for obtaining a smaller family of observables. One may conclude that qubit and observable resources seem to be linked. 


\section{CONCLUSION}

In [6], we introduced a universal set of projective measurements, which is minimal in terms of multi-qubit measurements and in terms of the number of needed ancillary qubits. In this paper, we prove that this set is not minimal in terms of one-qubit measurements, by introducing a universal set with only two one-qubit measurements. However, an additional ancillary qubit seems to be required. This leads to two open questions: is the set $\left\{Z, \frac{1}{\sqrt{2}}(X-Y), Z \otimes X\right\}$ minimal

in terms of one-qubit measurements? and is the minimal number of ancillary qubits with the set $\left\{Z, \frac{1}{\sqrt{2}}(X-Y), Z \otimes X\right\}$ to be one or two?

\section{REFERENCES}

1. S. A. Fenner and Y. Zhang. Universal quantum computation with two- and three-qubit projective measurements, arXiv.org report quant-ph/0111077, 2001.

2. A. Y. Kitaev, A. H. Shen and M. N. Vyalyi. Classical and Quantum Computation, American Mathematical Society, 2002.

3. M. A. Nielsen. Universal quantum computation using only projective measurement, quantum memory, and preparation of the O state, arXiv.org report quant-ph/0108020, 2001.

4. D. W. Leung. Two-qubit projective measurements are universal for quantum computation, arXiv.org report quant-ph/0111122, 2001.

5. D. W. Leung. Quantum computation by measurements, arXiv.org report quant-ph/0310189, 2003.

6. S. Perdrix State Transfer instead of Teleportation in Measurement-based Quantum Computation, arXiv.org report quantph/0402204, 2004.

7. S. Perdrix and Ph. Jorrand. Measurement-based Quantum Turing Machines and Questions of Universalities, arXiv.org report quant-ph/0402156, 2004. 